\title{
Recovery the interior temperature of a nonhomogeneous elliptic equation from boundary data
}

\author{
Tuan H Nguyen ${ }^{1 *}$ and Binh Thanh Tran²
}

\author{
*Correspondence: \\ nguyenhuytuan@tdt.edu.vn \\ ${ }^{1}$ Faculty of Mathematics and \\ Statistics, Ton Duc Thang University, \\ No. 19 Nguyen Huu Tho Street, Tan \\ Phong Ward, District 7, Ho Chi Minh, \\ Vietnam \\ Full list of author information is \\ available at the end of the article
}

\begin{abstract}
We consider the problem of finding a function $u$ from the boundary data $u(x, 1)$ and $u_{y}(x, 1)$, satisfying a nonhomogeneous elliptic equation

$$
\Delta u=f(x, y), \quad x \in \mathbb{R}, 0<y<1 .
$$

The problem is shown to be ill-posed. In this paper, we apply the Fourier transform to get an integral equation and give a regularized solution by directly perturbing this equation in combination with truncating high frequencies. The error estimate between the regularization solution and the exact solution is established. Finally, we present a numerical result which shows the effectiveness of the proposed method.
\end{abstract} MSC: $31 \mathrm{~A} 25 ; 34 \mathrm{~K} 29 ; 35 \mathrm{~J} 05$; 35J25; 35J99; 42A38; 44A35

Keywords: Fourier transform; ill-posed problem; quasi-boundary value methods; truncation method

\section{Introduction}

In this paper, we consider a problem of recovering the interior temperature from surface data (or boundary data). In fact, the interior temperature of a body (e.g., the skin of a missile) cannot be determined in several engineering contexts (see, e.g., [1-5]) and many industrial applications. Hence, in order to get the distribution of interior temperature, we have to use the measured temperature outside the surface. In optoelectronics, the determination of a radiation field surrounding a source of radiation (e.g., a light emitting diode) is a frequently occurring problem. As a rule, experimental determination of the whole radiation field is not possible. Practically, we are able to measure the electromagnetic field only on some subset of physical space (e.g., on some surfaces). So, the problem arises how to reconstruct the radiation field from such experimental data (see, for instance, [6]). In the paper of Reginska [6], the authors considered a physical problem which is connected with the notion of light beams. Some applications of this model can be established in more detail in [6].

Precisely, we consider a two-dimensional body represented by the domain $\mathbb{R} \times(0,1)$. Let $u(x, y)$ be the temperature of the body at $(x, y) \in \mathbb{R} \times(0,1)$, and let $f \equiv f(x, y)$ be a given source, we have the following nonhomogeneous equation:

$$
\Delta u=f(x, y), \quad x \in \mathbb{R}, 0<y<1
$$

○2014 Nguyen and Tran; licensee Springer. This is an Open Access article distributed under the terms of the Creative Commons Attribution License (http://creativecommons.org/licenses/by/2.0), which permits unrestricted use, distribution, and reproduction in any medium, provided the original work is properly cited. 
where $\Delta=\frac{\partial^{2}}{\partial x^{2}}+\frac{\partial^{2}}{\partial y^{2}}$. We assume that the temperature on the line $y=1$ is known, i.e.,

$$
u(x, 1)=\varphi(x)
$$

and that

$$
\frac{\partial u}{\partial y}(x, 1)=\psi(x)
$$

where $\varphi(x), \psi(x)$ are given functions in $L^{2}(\mathbb{R})$. The problem can be referred to as a sideways elliptic problem and the interior measurement $\varphi(x)$ is also called (in geology) the borehole measurement.

The latter problem is a Cauchy elliptic problem in an infinite strip and is well known as an ill-posed problem, i.e., solutions of the problem do not always exist and, whenever they do exist, there is no continuous dependence on the given data. This makes the numerical computations become difficult. So, ill-posed problems need to be regularized.

The homogeneous problem $(f \equiv 0)$ was studied with various methods in many papers. Using the boundary element method, the homogeneous problems were considered in [2, $7,8]$ etc. Similarly, many methods have been investigated to solve the Cauchy problem for a linear homogeneous elliptic equation such as the method of successive iterations $[8,9]$, the optimization method $[10,11]$, the quasi-reversibility method [12-14], fourthorder modified method $[15,16]$, Fourier truncation regularized (or spectral regularized method) [17-19], etc. The number of papers devoted to the Cauchy problem for linear homogeneous elliptic equation are very rich, for example, [7, 20-23] and the references therein.

Although there are many papers on homogeneous cases, we only find a few papers on nonhomogeneous sideways problems (for both parabolic and elliptic equations). The main aim of this paper is to present a simple and effective regularization method, and investigate the error estimate between the regularization solution and the exact solution. In a sense, this paper is an extension of recent results in [4, 9-11, 22, 24].

The paper is organized as follows. In Section 2, we present the formulation of the Cauchy problem for the elliptic equation and propose a modified regularization method. The error estimate is given based on two different a priori assumptions for the exact solution. Finally, in Section 3, we give a numerical example to demonstrate the effectiveness of our proposed method.

\section{Regularization and error estimate}

Let $\widehat{f}(\xi)=\frac{1}{\sqrt{2 \pi}} \int_{-\infty}^{+\infty} f(x) e^{-i \xi x} d x$ be the Fourier transform of function $f \in L^{2}(\mathbb{R})$. By taking Fourier transformation with respect to variable $x \in \mathbb{R}$, we transform problem (1)-(3) to the following form:

$$
\begin{aligned}
\widehat{u}(\xi, y)= & \frac{1}{2} \widehat{\varphi}(\xi)\left[e^{(1-y)|\xi|}+e^{(y-1)|\xi|}\right] \\
& +\frac{1}{2|\xi|} \widehat{\psi}(\xi)\left[e^{(y-1)|\xi|}-e^{(1-y)|\xi|}\right] \\
& +\frac{1}{2} \int_{y}^{1} \frac{1}{|\xi|}\left[e^{(\eta-y)|\xi|}-e^{-|y-\eta||\xi|}\right] \widehat{f}(\xi, \eta) d \eta
\end{aligned}
$$




$$
\begin{aligned}
= & \frac{1}{2} \widehat{\varphi}(\xi)\left[e^{(1-y)|\xi|}+e^{(y-1)|\xi|}\right]+\left[\frac{1-e^{2(1-y)|\xi|}}{2|\xi| e^{2(1-y)|\xi|}}\right] e^{(1-y)|\xi|} \widehat{\psi}(\xi) \\
& +\int_{y}^{1}\left[\frac{e^{2(\eta-y)|\xi|}-1}{2|\xi| e^{2(\eta-y)|\xi|}}\right] e^{(\eta-y) \mid \xi} \widehat{f}(\xi, \eta) d \eta .
\end{aligned}
$$

In the present paper, by approximating (4), we have a regularized solution $u_{\epsilon}(x, y)$, the Fourier transform of which is

$$
\begin{aligned}
\widehat{u}_{\epsilon}(\xi, y)= & {\left[\frac{1}{2} \widehat{\varphi}(\xi)\left(\frac{1}{\alpha(\epsilon)+e^{(y-1)|\xi|}}+e^{(y-1)|\xi|}\right)\right.} \\
& +\left(\frac{1-e^{2(1-y)|\xi|}}{2|\xi| e^{2(1-y)|\xi|}}\right) \frac{\widehat{\psi}(\xi)}{\alpha(\epsilon)+e^{(y-1)|\xi|}} \\
& \left.+\int_{y}^{1}\left(\frac{e^{2(\eta-y)|\xi|}-1}{2|\xi| e^{2(\eta-y)|\xi|}}\right) \frac{\widehat{f}(\xi, \eta)}{\alpha(\epsilon)+e^{(y-\eta)|\xi|}} d \eta\right] \chi_{[-\beta(\epsilon), \beta(\epsilon)]}(\xi)
\end{aligned}
$$

or

$$
\begin{aligned}
u_{\epsilon}(x, y)= & \frac{1}{2 \sqrt{2 \pi}} \int_{-\infty}^{+\infty}\left[\frac{1}{2} \widehat{\varphi}(\xi)\left(\frac{1}{\alpha(\epsilon)+e^{(y-1)|\xi|}}+e^{(y-1)|\xi|}\right)\right. \\
& +\left(\frac{1-e^{2(1-y)|\xi|}}{2|\xi| e^{2(1-y)|\xi|}}\right) \frac{\widehat{\psi}(\xi)}{\alpha(\epsilon)+e^{(y-1)|\xi|}} \\
& \left.+\int_{y}^{1}\left(\frac{e^{2(\eta-y)|\xi|}-1}{2|\xi| e^{2(\eta-y)|\xi|}}\right) \frac{\widehat{f}(\xi, \eta)}{\alpha(\epsilon)+e^{(y-\eta)|\xi|}} d \eta\right] \chi_{[-\beta(\epsilon), \beta(\epsilon)]}(\xi) e^{i \xi x} d \xi
\end{aligned}
$$

Here, $\alpha(\epsilon)$ and $\beta(\epsilon)$ are positive numbers (called regularization parameters) which depend on $\epsilon$. They will be chosen later such that $\alpha(\epsilon) \in(0,1)$ and $\beta(\epsilon) \rightarrow+\infty$ when $\epsilon \rightarrow 0$. For convenience, from now on, we denote $\alpha(\epsilon)$ by $\alpha$, and $\beta(\epsilon)$ by $\beta$.

In practice, the exact data $\left(\varphi_{e x}, \psi_{e x}\right) \in L^{2}(\mathbb{R}) \times L^{2}(\mathbb{R})$ is given only by measurement. Assume that the exact data $\left(\varphi_{e x}, \psi_{e x}\right)$ and the noisy data $\left(\varphi_{e x}, \psi_{e x}\right)$ both belong to $L^{2}(\mathbb{R}) \times$ $L^{2}(\mathbb{R})$ and satisfy the following noise level

$$
\left\|\varphi_{\epsilon}-\varphi_{e x}\right\|_{2} \leq \epsilon, \quad\left\|\psi_{\epsilon}-\psi_{e x}\right\|_{2} \leq \epsilon
$$

Let $v_{e x}$ and $v_{\epsilon}$ be the solutions of problem (6) corresponding to the exact data $\left(\varphi_{e x}, \psi_{e x}\right)$ and the measured data $\left(\varphi_{\epsilon}, \psi_{\epsilon}\right)$, respectively. Here, we denote by $\|\cdot\|_{2}$ the norm on $L^{2}(\mathbb{R})$. By taking the Fourier transform of $v_{e x}$ and $v_{\epsilon}$, we have

$$
\begin{aligned}
\widehat{v}_{e x}(\xi, y)= & {\left[\frac{1}{2} \widehat{\varphi}_{e x}(\xi)\left(\frac{1}{\alpha+e^{(y-1)|\xi|}}+e^{(y-1)|\xi|}\right)\right.} \\
& +\left(\frac{1-e^{2(1-y)|\xi|}}{2|\xi| e^{2(1-y)|\xi|}}\right) \frac{\widehat{\psi}_{e x}(\xi)}{\alpha+e^{(y-1)|\xi|}} \\
& \left.+\int_{y}^{1}\left(\frac{e^{2(\eta-y)|\xi|}-1}{2|\xi| e^{2(\eta-y)|\xi|}}\right) \frac{\widehat{f}(\xi, \eta)}{\alpha+e^{(y-\eta)|\xi|}} d \eta\right] \chi_{[-\beta, \beta]}(\xi),
\end{aligned}
$$




$$
\begin{aligned}
\widehat{v}_{\epsilon}(\xi, y)= & {\left[\frac{1}{2} \widehat{\varphi}_{\epsilon}(\xi)\left(\frac{1}{\alpha+e^{(y-1)|\xi|}}+e^{(y-1)|\xi|}\right)\right.} \\
& +\left(\frac{1-e^{2(1-y)|\xi|}}{2|\xi| e^{2(1-y)|\xi|}}\right) \frac{\widehat{\psi}_{\epsilon}(\xi)}{\alpha+e^{(y-1)|\xi|}} \\
& \left.+\int_{y}^{1}\left(\frac{e^{2(\eta-y)|\xi|}-1}{2|\xi| e^{2(\eta-y)|\xi|}}\right) \frac{\widehat{f}(\xi, \eta)}{\alpha+e^{(y-\eta)|\xi|}} d \eta\right] \chi_{[-\beta, \beta]}(\xi) .
\end{aligned}
$$

We first have the following lemma.

Lemma 1 (The stability of a solution of problem (5)) Suppose that $\varphi_{e x}, \psi_{e x}, \varphi_{\epsilon}, \psi_{\epsilon} \in L^{2}(\mathbb{R})$ and $\left\|\varphi_{\epsilon}-\varphi_{e x}\right\|_{2} \leq \epsilon,\left\|\psi_{\epsilon}-\psi_{e x}\right\|_{2} \leq \epsilon$. Then we have

$$
\left\|\widehat{v}_{\epsilon}(\cdot, y)-\widehat{v}_{e x}(\cdot, y)\right\|_{2} \leq \frac{2 \sqrt{2}}{\alpha(\epsilon)} \epsilon
$$

for all $y \in[0,1)$.

Proof From (7) and (8), we have

$$
\begin{aligned}
\left\|\widehat{v}_{\epsilon}(\cdot, y)-\widehat{v}_{e x}(\cdot, y)\right\|_{2}^{2} \\
=\int_{-\infty}^{+\infty}\left|\widehat{v}_{\epsilon}(\xi, y)-\widehat{v}_{e x}(\xi, y)\right|^{2} d \xi \\
=\int_{-\infty}^{+\infty}\left|\widehat{v}_{\epsilon}(\xi, y)-\widehat{v}_{e x}(\xi, y)\right|^{2} \chi_{[-\beta, \beta]}(\xi) d \xi \\
=\int_{-\infty}^{+\infty} \mid \frac{1}{2}\left(\frac{1}{\alpha+e^{-(y-1)|\xi|}}+e^{(y-1)|\xi|}\right)\left[\widehat{\varphi}_{\epsilon}(\xi)-\widehat{\varphi}_{e x}(\xi)\right] \\
\quad+\left.\left(\frac{1-e^{2(1-y)|\xi|}}{2|\xi| e^{2(1-y)|\xi|}}\right) \frac{1}{\alpha+e^{(y-1)|\xi|}}\left[\widehat{\psi}_{\epsilon}(\xi)-\widehat{\psi}_{e x}(\xi)\right]\right|^{2} \chi_{[-\beta, \beta]}(\xi) d \xi .
\end{aligned}
$$

Using the inequality $\frac{e^{|x|}-1}{|x|} \leq e^{|x|}$, we obtain

$$
\left|\frac{1-e^{2(1-y)|\xi|}}{2|\xi| e^{2(1-y)|\xi|}}\right| \leq 1 \quad \text { for } 0 \leq y<1 .
$$

Note that $\alpha(\epsilon) \in(0,1), e^{(y-1)|\xi|} \leq 1$ for $0<y<1$, then from (9) and (10) we get

$$
\begin{aligned}
& \left\|\widehat{v}_{\epsilon}(\cdot, y)-\widehat{v}_{e x}(\cdot, y)\right\|_{2}^{2} \\
& \quad \leq \int_{-\infty}^{+\infty}\left[\frac{1}{2}\left(\frac{1}{\alpha}+1\right)\left|\widehat{\varphi}_{\epsilon}(\xi)-\widehat{\varphi}_{e x}(\xi)\right|+\frac{1}{\alpha}\left|\widehat{\psi}_{\epsilon}(\xi)-\widehat{\psi}_{e x}(\xi)\right|\right]^{2} d \xi \\
& \quad \leq \frac{1}{\alpha^{2}} \int_{-\infty}^{+\infty}\left(\left|\widehat{\varphi}_{\epsilon}(\xi)-\widehat{\varphi}_{e x}(\xi)\right|+\left|\widehat{\psi}_{\epsilon}(\xi)-\widehat{\psi}_{e x}(\xi)\right|\right)^{2} d \xi \\
& \quad \leq \frac{2}{\alpha^{2}} \int_{-\infty}^{+\infty}\left(\left|\widehat{\varphi}_{\epsilon}(\xi)-\widehat{\varphi}_{e x}(\xi)\right|^{2}+\left|\widehat{\psi}_{\epsilon}(\xi)-\widehat{\psi}_{e x}(\xi)\right|^{2}\right) d \xi \\
& \quad=\frac{2}{\alpha^{2}}\left(\left\|\widehat{\varphi}_{\epsilon}-\widehat{\varphi}_{e x}\right\|_{2}^{2}+\left\|\widehat{\psi}_{\epsilon}-\widehat{\psi}_{e x}\right\|_{2}^{2}\right) .
\end{aligned}
$$


Applying the inequality $\sqrt{a^{2}+b^{2}} \leq a+b$ for $a, b \geq 0$, we obtain

$$
\begin{aligned}
\left\|\widehat{v}_{\epsilon}(\cdot, y)-\widehat{v}_{e x}(\cdot, y)\right\|_{2} & \leq \frac{\sqrt{2}}{\alpha^{2}}\left(\left\|\widehat{\varphi}_{\epsilon}-\widehat{\varphi}_{e x}\right\|_{2}+\left\|\widehat{\psi}_{\epsilon}-\widehat{\psi}_{e x}\right\|_{2}\right) \\
& \leq \frac{2 \sqrt{2}}{\alpha} \epsilon .
\end{aligned}
$$

This completes the proof of Lemma 1.

Theorem 1 Assume that $u_{e x}$ is the exact solution of problem (1)-(3) corresponding to the exact data $\varphi_{e x}, \psi_{e x} \in L^{2}(\mathbb{R})$ and that $\varphi_{\epsilon}, \psi_{\epsilon} \in L^{2}(\mathbb{R})$ are the measured data satisfying $\| \varphi_{\epsilon}-$ $\varphi_{e x}\left\|_{2} \leq \epsilon,\right\| \psi_{\epsilon}-\psi_{e x} \|_{2} \leq \epsilon$. Moreover, if we assume in addition that $\frac{\partial}{\partial x} u_{e x} \in L^{2}(\mathbb{R})$ and $\left.\int_{0}^{1} \int_{-\infty}^{+\infty} \widehat{f}(\xi, \eta)\right|^{2} d \xi d \eta<\infty$. Then, with $\alpha=\epsilon^{1 / 2}$ and $\beta=\ln \frac{1}{\epsilon^{1 / 8}}$, we can construct from $\varphi_{\epsilon}$, $\psi_{\epsilon}$ a function $v_{\epsilon} \in L^{2}(\mathbb{R})$ for every $0 \leq y<1$

$$
\left\|v_{\epsilon}(\cdot, y)-u_{e x}(\cdot, y)\right\|_{2} \leq \frac{C}{\ln \frac{1}{\epsilon}}
$$

where

$$
\begin{aligned}
& C=2 \sqrt{2}+\sqrt{3} M+8\left\|\frac{\partial}{\partial x} u_{e x}(\cdot, y)\right\|_{2} \\
& M=\frac{1}{2}\left\|\widehat{\varphi}_{e x}(\xi)\right\|_{2}+\left\|\widehat{\psi}_{e x}(\xi)\right\|_{2}+\sqrt{\int_{0}^{1} \int_{-\infty}^{+\infty}|\widehat{f}(\xi, \eta)|^{2} d \xi d \eta .}
\end{aligned}
$$

Proof From (4), we have

$$
\begin{aligned}
\widehat{u}_{e x}(\xi, y)= & \frac{1}{2} \widehat{\varphi}_{e x}(\xi)\left(e^{(1-y)|\xi|}+e^{(y-1)|\xi|}\right)+\left(\frac{1-e^{2(1-y)|\xi|}}{2|\xi| e^{2(1-y)|\xi|}}\right) e^{(1-y)|\xi|} \widehat{\psi}_{e x}(\xi) \\
& +\int_{y}^{1}\left(\frac{e^{2(\eta-y)|\xi|}-1}{2|\xi| e^{2(\eta-y)|\xi|}}\right) e^{(\eta-y) \mid \xi} \widehat{f}(\xi, \eta) d \eta .
\end{aligned}
$$

Taking into account (7) and (11), we get

$$
\begin{aligned}
\left\|\widehat{v}_{e x}(\cdot, y)-\widehat{u}_{e x}(\cdot, y)\right\|_{2}^{2} \\
=\int_{-\infty}^{+\infty}\left|\widehat{v}_{e x}(\xi, y)-\widehat{u}_{e x}(\xi, y)\right|^{2} d \xi \\
=\int_{-\infty}^{+\infty}\left|\widehat{v}_{e x}(\xi, y)-\widehat{u}_{e x}(\xi, y)\right|^{2} \chi_{[-\beta, \beta]}(\xi) d \xi \\
\quad+\int_{-\infty}^{+\infty}\left|\widehat{v}_{e x}(\xi, y)-\widehat{u}_{e x}(\xi, y)\right|^{2} \chi_{(-\infty,-\beta) \cup(\beta,+\infty)}(\xi) d \xi \\
=\int_{-\infty}^{+\infty}\left|\widehat{v}_{e x}(\xi, y)-\widehat{u}_{e x}(\xi, y)\right|^{2} \chi_{[-\beta, \beta]}(\xi) d \xi \\
\quad+\int_{-\infty}^{+\infty}\left|\widehat{u}_{e x}(\xi, y)\right|^{2} \chi_{(-\infty,-\beta) \cup(\beta,+\infty)}(\xi) d \xi .
\end{aligned}
$$


Moreover, one has, for $0 \leq y<1$,

$$
\left|\frac{1}{\alpha+e^{(y-1)|\xi|}}-e^{(1-y)|\xi|}\right| \leq \alpha e^{2|\xi|}
$$

By a similar way, we also have

$$
\left|\frac{1}{\alpha+e^{(y-\eta)|\xi|}}-e^{(\eta-y)|\xi|}\right| \leq \alpha e^{2|\xi|} \quad \text { for } 0<y<\eta<1 .
$$

Moreover, using the inequality $\frac{e^{|x|}-1}{|x|} \leq e^{|x|}$, we have

$$
\left|\frac{1-e^{2(\eta-y)|\xi|}}{2|\xi| e^{2(\eta-y)|\xi|}}\right| \leq 1 \quad \text { for } 0<y<\eta<1
$$

From (7), (11), (13), (14), (15), we obtain

$$
\begin{aligned}
& \left\|\widehat{v}_{e x}(\cdot, y)-\widehat{u}_{e x}(\cdot, y)\right\|_{2}^{2} \\
& \leq \int_{-\infty}^{+\infty}\left(\frac{1}{2} \alpha e^{2|\xi|}\left|\widehat{\varphi}_{e x}(\xi)\right|+\alpha e^{2|\xi|}\left|\widehat{\psi}_{e x}(\xi)\right|+\alpha e^{2|\xi|} \int_{0}^{1}|\widehat{f}(\xi, \eta)| d \eta\right)^{2} \chi_{[-\beta, \beta]}(\xi) d \xi \\
& \quad+\int_{-\infty}^{+\infty} \frac{1}{|\xi|^{2}}\left|\xi \widehat{u}_{e x}(\xi, y)\right|^{2} \chi_{(-\infty,-\beta) \cup(\beta,+\infty)}(\xi) d \xi
\end{aligned}
$$

It follows from $(a+b+c)^{2} \leq 3\left(a^{2}+b^{2}+c^{2}\right)$ that

$$
\begin{aligned}
&\left\|\widehat{v}_{e x}(\cdot, y)-\widehat{u}_{e x}(\cdot, y)\right\|_{2}^{2} \\
& \leq \frac{1}{4} \alpha^{2} \int_{-\infty}^{+\infty} e^{4|\xi|}\left|\widehat{\varphi}_{e x}(\xi)\right|^{2} \chi_{[-\beta, \beta]}(\xi) d \xi \\
&+\alpha^{2} \int_{-\infty}^{+\infty} e^{4|\xi|}\left|\widehat{\psi}_{e x}(\xi)\right|^{2} \chi_{[-\beta, \beta]}(\xi) d \xi \\
&+\alpha^{2} \int_{-\infty}^{+\infty}\left(\int_{0}^{1}|\widehat{f}(\xi, \eta)| d \eta\right)^{2} e^{2|\xi|} \chi_{[-\beta, \beta]}(\xi) d \xi \\
&+\int_{-\infty}^{+\infty} \frac{1}{|\xi|^{2}}\left|\xi \widehat{u}_{e x}(\xi, y)\right|^{2} \chi_{(-\infty,-\beta) \cup(\beta,+\infty)}(\xi) d \xi \\
& \leq \frac{3}{4} \alpha^{2} e^{4 \beta} \int_{-\infty}^{+\infty}\left|\widehat{\varphi}_{e x}(\xi)\right|^{2} \chi_{[-\beta, \beta]}(\xi) d \xi+3 \alpha^{2} e^{4 \beta} \int_{-\infty}^{+\infty}\left|\widehat{\psi}_{e x}(\xi)\right|^{2} \chi_{[-\beta, \beta]}(\xi) d \xi \\
&+3 \alpha^{2} e^{4 \beta} \int_{-\infty}^{+\infty}\left(\int_{0}^{1}|\widehat{f}(\xi, \eta)|^{2} d \eta\right) \chi_{[-\beta, \beta]}(\xi) d \xi \\
&+\frac{1}{\beta^{2}} \int_{-\infty}^{+\infty}\left|\xi \widehat{u}_{e x}(\xi, y)\right|^{2} \chi_{(-\infty,-\beta) \cup(\beta,+\infty)}(\xi) d \xi \\
& \leq \frac{3}{4} \alpha^{2} e^{4 \beta}\left\|\widehat{\varphi}_{e x}(\xi)\right\|_{2}^{2}+3 \alpha^{2} e^{4 \beta}\left\|\widehat{\psi}_{e x}(\xi)\right\|_{2}^{2} \\
&+3 \alpha^{2} e^{4 \beta} \int_{0}^{1} \int_{-\infty}^{+\infty}|\widehat{f}(\xi, \eta)|^{2} d \xi d \eta+\frac{1}{\beta^{2}}\left\|\frac{\partial}{\partial x} u_{e x}(\cdot, y)\right\|_{2}^{2}
\end{aligned}
$$


Applying the inequality $\sqrt{a^{2}+b^{2}+c^{2}+d^{2}} \leq a+b+c+d$ for $a, b, c, d \geq 0$, we obtain

$$
\begin{aligned}
& \left\|\widehat{v}_{e x}(\cdot, y)-\widehat{u}_{e x}(\cdot, y)\right\|_{2} \\
& \leq \quad \sqrt{3} \alpha e^{2 \beta}\left(\frac{1}{2}\left\|\widehat{\varphi}_{e x}(\xi)\right\|_{2}+\left\|\widehat{\psi}_{e x}(\xi)\right\|_{2}+\sqrt{\left.\int_{0}^{1} \int_{-\infty}^{+\infty}|\widehat{f}(\xi, \eta)|^{2} d \xi d \eta\right)}\right. \\
& \quad+\frac{1}{\beta}\left\|\frac{\partial}{\partial x} u_{e x}(\cdot, y)\right\|_{2} .
\end{aligned}
$$

According to the triangle inequality,

$$
\left\|\widehat{v}_{\epsilon}(\cdot, y)-\widehat{u}_{e x}(\cdot, y)\right\|_{2} \leq\left\|\widehat{v}_{\epsilon}(\cdot, y)-\widehat{v}_{e x}(\cdot, y)\right\|_{2}+\left\|\widehat{v}_{e x}(\cdot, y)-\widehat{u}_{e x}(\cdot, y)\right\|_{2}
$$

so using Parseval's equality, (16) and Lemma 1, we get

$$
\begin{aligned}
& \left\|v_{\epsilon}(\cdot, y)-u_{e x}(\cdot, y)\right\|_{2} \\
& \leq \frac{2 \sqrt{2}}{\alpha} \epsilon+\sqrt{3} \alpha e^{2 \beta}\left(\frac{1}{2}\left\|\widehat{\varphi}_{e x}(\xi)\right\|_{2}+\left\|\widehat{\psi}_{e x}(\xi)\right\|_{2}+\sqrt{\int_{0}^{1} \int_{-\infty}^{+\infty}|\widehat{f}(\xi, \eta)|^{2} d \xi d \eta}\right) \\
& \quad+\frac{1}{\beta}\left\|\frac{\partial}{\partial x} u_{e x}(\cdot, y)\right\|_{2}
\end{aligned}
$$

The choice of $\alpha=\epsilon^{1 / 2}$ and $\beta=\ln \frac{1}{\epsilon^{1 / 8}}$ leads to

$$
\left\|v_{\epsilon}(\cdot, y)-u_{e x}(\cdot, y)\right\|_{2} \leq \frac{C}{\ln \frac{1}{\epsilon}}
$$

where

$$
\begin{aligned}
& C=2 \sqrt{2}+\sqrt{3} M+8\left\|\frac{\partial}{\partial x} u_{e x}(\cdot, y)\right\|_{2} \\
& M=\frac{1}{2}\left\|\widehat{\varphi}_{e x}(\xi)\right\|_{2}+\left\|\widehat{\psi}_{e x}(\xi)\right\|_{2}+\sqrt{\int_{0}^{1} \int_{-\infty}^{+\infty}|\widehat{f}(\xi, \eta)|^{2} d \xi d \eta .}
\end{aligned}
$$

This completes the proof of Theorem 1 .

Remark Theorem 1 gives a good approximation not only in the case $0<y<1$ but also in the case $y=0$.

If we choose $\alpha=\epsilon^{k}, 0<k<1$, and $e^{2 \beta} \alpha=\epsilon^{m}, m<k$, then $\beta=\sqrt{\frac{1}{\epsilon^{k-m}}}$ and

$$
\begin{aligned}
& \left\|v_{\epsilon}(\cdot, y)-u_{e x}(\cdot, y)\right\|_{2} \\
& \leq 2 \sqrt{2} \epsilon^{1-k}+\sqrt{3} \epsilon^{m}\left(\frac{1}{2}\left\|\widehat{\varphi}_{e x}(\xi)\right\|_{2}+\left\|\widehat{\psi}_{e x}(\xi)\right\|_{2}+\sqrt{\int_{0}^{1} \int_{-\infty}^{+\infty}|\widehat{f}(\xi, \eta)|^{2} d \xi d \eta}\right) \\
& \quad+\sqrt{\epsilon^{k-m}}\left\|\frac{\partial}{\partial x} u_{e x}(\cdot, y)\right\|_{2} .
\end{aligned}
$$

Let $k=\frac{1}{2}$ and $m=\frac{1}{4}$, we have Theorem 1 . 


\section{Numerical experiment}

In this section, we present a simple example intended to demonstrate the usefulness of the approach. The test was performed using Matlab 6.1. The numerical example was constructed in the following way: first we selected the initial data $u(x, 1)=\varphi(x)$ and $\frac{\partial u}{\partial y}(x, 1)=$ $\psi(x)$. We have the following problem

$$
\Delta u=f(x, y), \quad x \in \mathbb{R}, 0<y<1,
$$

where $u$ satisfies

$$
\begin{aligned}
& u(x, 1)=\varphi(x), \\
& \frac{\partial u}{\partial y}(x, 1)=\psi(x) .
\end{aligned}
$$

Let $\varphi^{\epsilon}(x, y), \psi^{\epsilon}$ be the disturbed measure data such that $\left\|\varphi(x)-\varphi^{\epsilon}(x)\right\| \leq \epsilon, \| \psi(x)-$ $\psi^{\epsilon}(x) \| \leq \epsilon$.

For example, we take

$$
\begin{aligned}
& f(x, y)=-4\left(1-x^{2}-y^{2}\right) e^{-x^{2}-y^{2}}, \\
& \varphi(x)=e^{-x^{2}-1} \\
& \psi(x)=-2 e^{-x^{2}-1}, \\
& \varphi^{\epsilon}(x)=\varphi(x)+\epsilon \cdot \operatorname{rand}(), \\
& \psi^{\epsilon}(x)=\psi(x)+\epsilon \cdot \operatorname{rand}() .
\end{aligned}
$$

In the numerical experiment, we always fix the interval $0 \leq x \leq 3$.

For an exact data function $\varphi(x)$, its discrete noisy version is

$$
\varphi^{\epsilon}(x)=\varphi(x)+\epsilon \cdot \operatorname{rand}()
$$

where

$$
\varphi=\left(\varphi\left(x_{1}\right), \ldots, \varphi\left(x_{N}\right)\right), \quad x_{j}=-3+\frac{3(j-1)}{N-1}, j=1,2, \ldots, N,
$$

and

$$
\epsilon=\left\|\varphi^{\epsilon}-\varphi\right\|=\sqrt{\frac{1}{N} \sum_{j=1}^{N}\left|\varphi\left(x_{j}\right)-\varphi^{\epsilon}\left(x_{j}\right)\right|^{2}} .
$$

The function rand( $\cdot)$ generates arrays of random numbers whose elements are normally distributed with mean 0 , variance $\sigma^{2}=1$, and $\operatorname{SD} \sigma=1$. N is the total test points at $\mathrm{x}$-axis. In our computations, we always take $N=100$. Let us define the error estimate $\delta^{i, \epsilon}$ between the exact solution $u(\cdot, y)$ and regularized solutions $u^{i, \epsilon}(\cdot, y)$ at given value $y$

$$
\delta^{i, \epsilon}(y)=\left\|u(\cdot, y)-u^{i, \epsilon}(\cdot, y)\right\| .
$$


Table 1 Error estimations for the first regularization solution $\left(\delta^{1, \epsilon}\right)$ and the second regularization solution $\left(\delta^{2, \epsilon}\right)$

\begin{tabular}{|c|c|c|c|c|c|c|c|c|}
\hline \multirow[t]{2}{*}{$y$} & \multicolumn{2}{|l|}{$\epsilon=10^{-1}$} & \multicolumn{2}{|l|}{$\epsilon=10^{-3}$} & \multicolumn{2}{|l|}{$\epsilon=10^{-5}$} & \multicolumn{2}{|l|}{$\epsilon=10^{-7}$} \\
\hline & $\overline{\delta^{1, \epsilon}}$ & $\delta^{2, \epsilon}$ & $\overline{\delta^{1, \epsilon}}$ & $\delta^{2, \epsilon}$ & $\overline{\delta^{1, \epsilon}}$ & $\delta^{2, \epsilon}$ & $\overline{\delta^{1, \epsilon}}$ & $\delta^{2, \epsilon}$ \\
\hline 0.0 & $2.570 \mathrm{E}-01$ & $1.035 \mathrm{E}+01$ & $2.147 \mathrm{E}-01$ & $1.903 \mathrm{E}+00$ & $2.147 \mathrm{E}-01$ & $2.987 \mathrm{E}-01$ & $2.147 \mathrm{E}-01$ & $2.158 \mathrm{E}-01$ \\
\hline 0.1 & $3.037 \mathrm{E}+00$ & $1.080 \mathrm{E}+01$ & $1.852 \mathrm{E}+00$ & $2.321 \mathrm{E}+00$ & $1.335 \mathrm{E}+00$ & $3.359 \mathrm{E}-01$ & $1.053 \mathrm{E}+00$ & $1.964 \mathrm{E}-01$ \\
\hline 0.2 & $5.033 E+00$ & $1.101 \mathrm{E}+01$ & $3.108 \mathrm{E}+00$ & $2.799 \mathrm{E}+00$ & $2.261 \mathrm{E}+00$ & $4.275 \mathrm{E}-01$ & $1.788 \mathrm{E}+00$ & $1.825 \mathrm{E}-01$ \\
\hline 0.3 & $6.252 \mathrm{E}+00$ & $1.098 \mathrm{E}+01$ & $3.954 \mathrm{E}+00$ & $3.289 \mathrm{E}+00$ & $2.910 \mathrm{E}+00$ & $5.756 \mathrm{E}-01$ & $2.316 \mathrm{E}+00$ & $1.902 \mathrm{E}-01$ \\
\hline 0.4 & $6.932 \mathrm{E}+00$ & $1.075 \mathrm{E}+01$ & $4.497 \mathrm{E}+00$ & $3.751 \mathrm{E}+00$ & $3.348 \mathrm{E}+00$ & $7.574 \mathrm{E}-01$ & $2.682 \mathrm{E}+00$ & $2.319 \mathrm{E}-01$ \\
\hline 0.5 & $7.236 \mathrm{E}+00$ & $1.037 \mathrm{E}+01$ & $4.815 \mathrm{E}+00$ & $4.156 \mathrm{E}+00$ & $3.624 \mathrm{E}+00$ & $4.868 \mathrm{E}-04$ & $2.920 \mathrm{E}+00$ & $2.954 \mathrm{E}-01$ \\
\hline 0.6 & $7.280 \mathrm{E}+00$ & $9.873 \mathrm{E}+00$ & $4.965 \mathrm{E}+00$ & $4.483 E+00$ & $3.775 \mathrm{E}+00$ & $9.559 \mathrm{E}-01$ & $3.061 \mathrm{E}+00$ & $3.703 \mathrm{E}-01$ \\
\hline 0.7 & $7.143 \mathrm{E}+00$ & $9.308 \mathrm{E}+00$ & $4.990 \mathrm{E}+00$ & $4.725 \mathrm{E}+00$ & $3.833 \mathrm{E}+00$ & $1.359 \mathrm{E}+00$ & $3.125 \mathrm{E}+00$ & $4.508 \mathrm{E}-01$ \\
\hline 0.8 & $6.885 \mathrm{E}+00$ & $8.702 \mathrm{E}+00$ & $4.925 \mathrm{E}+00$ & $4.877 \mathrm{E}+00$ & $3.818 \mathrm{E}+00$ & $1.548 \mathrm{E}+00$ & $3.131 \mathrm{E}+00$ & $5.327 \mathrm{E}-01$ \\
\hline 0.9 & $6.548 \mathrm{E}+00$ & $8.082 \mathrm{E}+00$ & $4.793 E+00$ & $4.943 E+00$ & $3.751 \mathrm{E}+00$ & $1.721 \mathrm{E}+00$ & $3.092 \mathrm{E}+00$ & $6.135 \mathrm{E}-01$ \\
\hline 1.0 & $6.162 \mathrm{E}+00$ & $7.464 \mathrm{E}+00$ & $4.615 \mathrm{E}+00$ & $4.930 \mathrm{E}+00$ & $3.644 \mathrm{E}+00$ & $1.874 \mathrm{E}+00$ & $3.019 \mathrm{E}+00$ & $6.910 \mathrm{E}-01$ \\
\hline
\end{tabular}


Table 2 Relative error estimations for the first method $\left(\sigma^{1, \epsilon}\right)$ and second regularization solution $\left(\sigma^{2, \epsilon}\right)$

\begin{tabular}{|c|c|c|c|c|c|c|c|c|}
\hline \multirow[t]{2}{*}{$y$} & \multicolumn{2}{|l|}{$\epsilon=10^{-1}$} & \multicolumn{2}{|l|}{$\epsilon=10^{-3}$} & \multicolumn{2}{|l|}{$\epsilon=10^{-5}$} & \multicolumn{2}{|l|}{$\epsilon=10^{-7}$} \\
\hline & $\overline{\delta^{1, \epsilon}}$ & $\delta^{2, \epsilon}$ & $\overline{\delta^{1, \epsilon}}$ & $\delta^{2, \epsilon}$ & $\overline{\delta^{1, \epsilon}}$ & $\delta^{2, \epsilon}$ & $\overline{\delta^{1, \epsilon}}$ & $\delta^{2, \epsilon}$ \\
\hline 0.0 & $1.174 \mathrm{E}-05$ & $2.111 \mathrm{E}-02$ & $7.783 \mathrm{E}-06$ & $7.117 \mathrm{E}-04$ & $7.782 \mathrm{E}-06$ & $1.619 \mathrm{E}-05$ & $7.782 \mathrm{E}-06$ & $7.862 \mathrm{E}-06$ \\
\hline 0.1 & $2.219 \mathrm{E}-03$ & $2.810 \mathrm{E}-02$ & $8.234 \mathrm{E}-04$ & $1.295 \mathrm{E}-03$ & $4.269 \mathrm{E}-04$ & $2.561 \mathrm{E}-05$ & $2.646 \mathrm{E}-04$ & $7.954 \mathrm{E}-06$ \\
\hline 0.2 & $7.447 \mathrm{E}-03$ & $3.563 \mathrm{E}-02$ & $2.838 \mathrm{E}-03$ & $2.302 \mathrm{E}-03$ & $1.501 \mathrm{E}-03$ & $5.188 \mathrm{E}-05$ & $9.381 \mathrm{E}-04$ & $8.393 \mathrm{E}-06$ \\
\hline 0.3 & $1.404 \mathrm{E}-02$ & $4.328 \mathrm{E}-02$ & $5.614 \mathrm{E}-03$ & $3.884 \mathrm{E}-03$ & $3.040 \mathrm{E}-03$ & $1.168 \mathrm{E}-04$ & $1.924 \mathrm{E}-03$ & $1.140 \mathrm{E}-05$ \\
\hline 0.4 & $2.108 \mathrm{E}-02$ & $5.068 \mathrm{E}-02$ & $8.872 \mathrm{E}-03$ & $6.172 \mathrm{E}-03$ & $4.915 \mathrm{E}-03$ & $2.491 \mathrm{E}-04$ & $3.152 \mathrm{E}-03$ & $2.175 \mathrm{E}-05$ \\
\hline 0.5 & $2.807 \mathrm{E}-02$ & $5.759 \mathrm{E}-02$ & $1.242 \mathrm{E}-02$ & $9.252 \mathrm{E}-03$ & $7.034 \mathrm{E}-03$ & $4.869 \mathrm{E}-04$ & $4.568 \mathrm{E}-03$ & $4.465 \mathrm{E}-05$ \\
\hline 0.6 & $3.469 \mathrm{E}-02$ & $6.381 \mathrm{E}-02$ & $1.614 \mathrm{E}-02$ & $1.316 \mathrm{E}-02$ & $9.328 \mathrm{E}-03$ & $8.769 \mathrm{E}-04$ & $6.131 \mathrm{E}-03$ & $8.743 \mathrm{E}-05$ \\
\hline 0.7 & $4.080 \mathrm{E}-02$ & $6.927 \mathrm{E}-02$ & $1.991 \mathrm{E}-02$ & $1.785 \mathrm{E}-02$ & $1.174 \mathrm{E}-02$ & $1.474 \mathrm{E}-03$ & $7.807 \mathrm{E}-03$ & $1.599 \mathrm{E}-04$ \\
\hline 0.8 & $4.630 \mathrm{E}-02$ & $7.396 \mathrm{E}-02$ & $2.368 \mathrm{E}-02$ & $2.323 \mathrm{E}-02$ & $1.424 \mathrm{E}-02$ & $2.337 \mathrm{E}-03$ & $9.571 \mathrm{E}-03$ & $2.744 \mathrm{E}-04$ \\
\hline 0.9 & $5.115 \mathrm{E}-02$ & $7.791 \mathrm{E}-02$ & $2.740 \mathrm{E}-02$ & $2.914 \mathrm{E}-02$ & $1.678 \mathrm{E}-02$ & $3.529 \mathrm{E}-03$ & $1.140 \mathrm{E}-02$ & $4.459 \mathrm{E}-04$ \\
\hline 1.0 & $5.532 \mathrm{E}-02$ & $8.118 \mathrm{E}-02$ & $3.103 \mathrm{E}-02$ & $3.541 \mathrm{E}-02$ & $1.934 \mathrm{E}-02$ & $5.111 \mathrm{E}-03$ & $1.328 \mathrm{E}-02$ & $6.925 \mathrm{E}-04$ \\
\hline
\end{tabular}




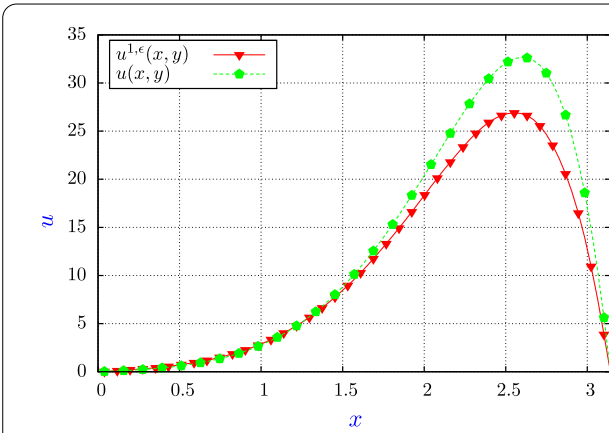

(a) $u^{1, \epsilon}(x, y), \quad \epsilon=10^{-1}$

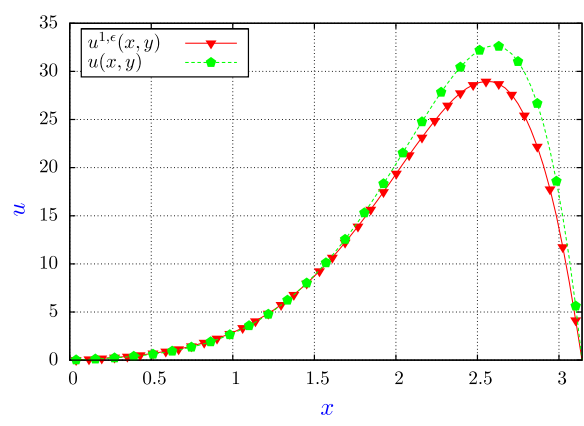

(c) $u^{1, \epsilon}(x, y), \quad \epsilon=10^{-3}$

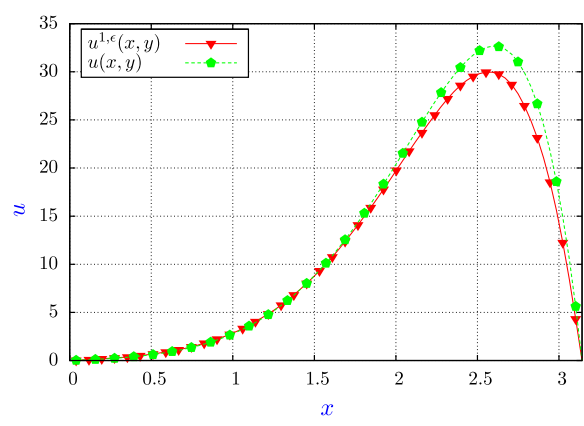

(e) $u^{1, \epsilon}(x, y), \quad \epsilon=10^{-5}$

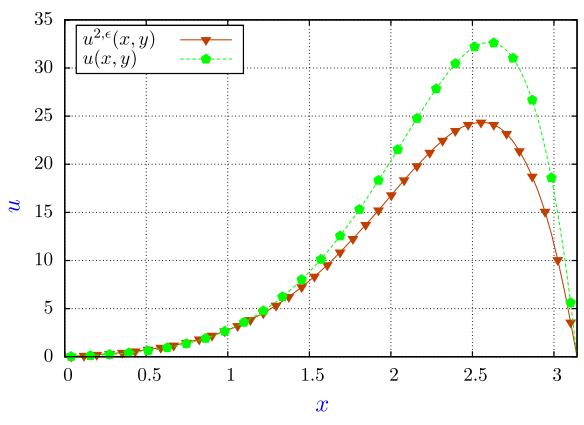

(b) $u^{2, \epsilon}(x, y), \quad \epsilon=10^{-1}$

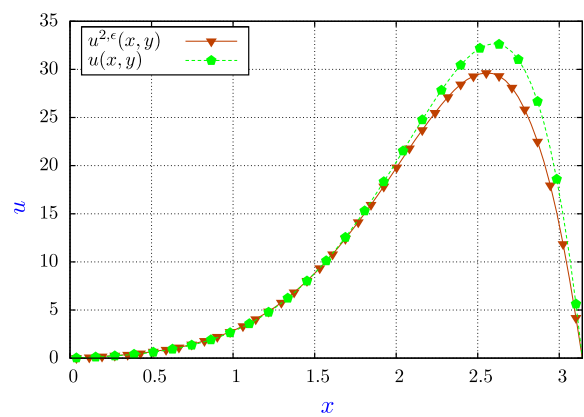

(d) $u^{2, \epsilon}(x, y), \quad \epsilon=10^{-3}$

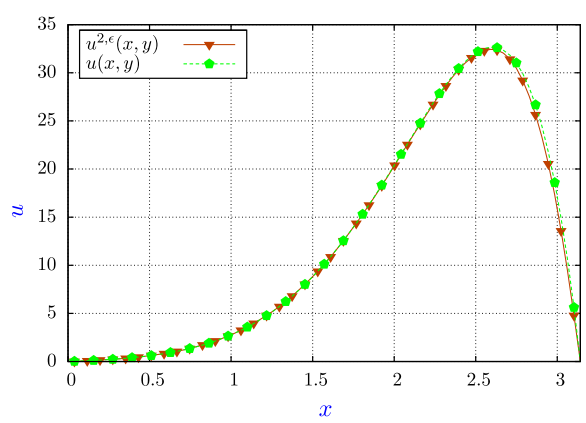

(f) $u^{2, \epsilon}(x, y), \quad \epsilon=10^{-5}$

Figure $12 \mathrm{D}$ graphs of section cut at $y=0.5$ of the exact solution $u(x, y)$ and regularized solutions $u^{i, \epsilon}(x, y)$.

Here, $u^{i, \epsilon}$ regularized solutions are calculated by the first regularization solution $(i=1)$ with $\alpha=\epsilon^{1 / 2}, \beta=\frac{-1}{\ln 8 \epsilon}$ and the second regularization $(i=2)$ with $\alpha=\epsilon^{1 / 2}, \beta=\frac{-1}{\ln 10 \epsilon}$.

Table 1 shows the error estimate between the exact solution and the regularized solutions. In the table, we see that when $\epsilon=10^{-1}$ the error estimations of the first method are slightly better than the error estimations of the second method. However, starting from $\epsilon=10^{-3}$, the second method gradually gives much better results.

Because the error estimations in $L_{2}$ are relatively big, it is difficult to see the effectiveness of our methods. For a better illustration, we define the relative error estimate between exact solution and regularized solutions as follows:

$$
\sigma^{i, \epsilon}(y)=\frac{\sqrt{\sum_{k=1}^{I}\left|u^{i, \epsilon}\left(x_{k}, y\right)-u\left(x_{k}, y\right)\right|^{2}}}{\sqrt{\sum_{k=1}^{I}\left|u\left(x_{k}, y\right)\right|^{2}}} .
$$




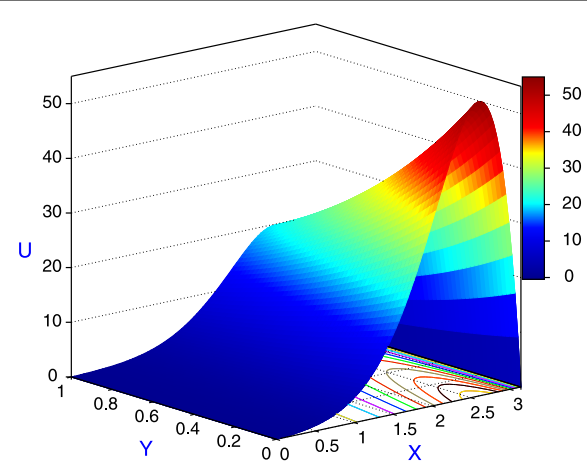

(a) Exact solution

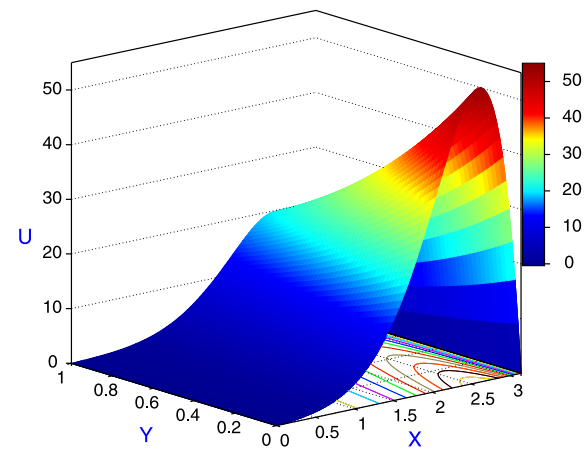

(b) $u^{1, \epsilon}(x, y), \quad \epsilon=10^{-1}$

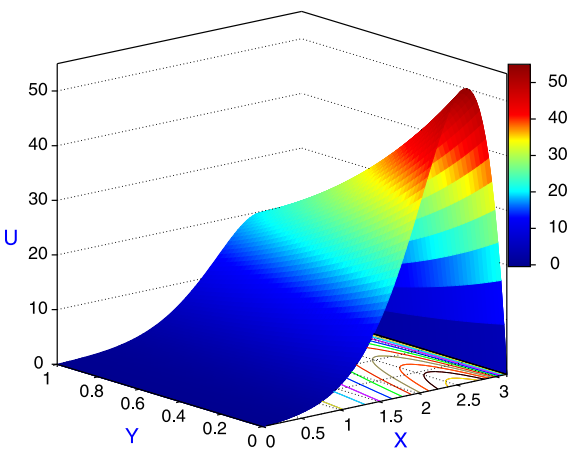

(c) $u^{2, \epsilon}(x, y), \quad \epsilon=10^{-1}$

Figure $23 D$ graphs of the exact solution $u(x, y)$ and regularized solutions $u^{i, \epsilon}(x, y)$.

Table 2 shows the relative error estimations between the exact solution and the first regularized solution $\left(\sigma^{1, \epsilon}\right)$ and by the second regularization solution $\left(\sigma^{2, \epsilon}\right)$. Obviously from the table, our proposed method is stable and effective.

We also present some graphical representations of the exact solution and regularized solutions. Figure 2 is the 3-D representation of these solutions, and Figure 1 shows some graphs of section cut at value $y=0.5$, with $\epsilon=10^{-i}, i=1,3,5$. From the graphs, we see that the second method gives very precise solution when $\epsilon=10^{-5}$.

\section{Competing interests}

The authors declare that they have no competing interests.

\section{Authors' contributions}

All authors contributed equally and significantly in writing this paper. All authors read and approved the final manuscript.

\section{Author details}

${ }^{1}$ Faculty of Mathematics and Statistics, Ton Duc Thang University, No. 19 Nguyen Huu Tho Street, Tan Phong Ward, District 7, Ho Chi Minh, Vietnam. ${ }^{2}$ Department of Mathematics and Applications, Sai Gon University, 273 An Duong Vuong, Q.5, Ho Chi Minh, Vietnam.

\section{Acknowledgements}

This project was supported by Ton Duc Thang University (FOSTECH). The authors would like to thank the anonymous referees for their valuable suggestions and comments leading to the improvement of our manuscript. We would like to thank Truong Trong Nghia in School of Computing, the University of Utah, USA for his most helpful comments on numerical results.

\section{Received: 16 August 2013 Accepted: 10 December 2013 Published: 14 Jan 2014}

\section{References}

1. Beskos, DE: Boundary element method in dynamic analysis: Part II (1986-1996). Appl. Mech. Rev. 50, 149-197 (1997) 
2. Chen, JT, Wong, FC: Dual formulation of multiple reciprocity method for the acoustic mode of a cavity with a thin partition. J. Sound Vib. 217, 7595 (1998)

3. Harari, I, Barbone, PE, Slavutin, M, Shalom, R: Boundary infinite elements for the Helmholtz equation in exterior domains. Int. J. Numer. Methods Eng. 41, 1105-1131 (1998)

4. Marin, L, Elliott, L, Heggs, PJ, Ingham, DB, Lesnic, D, Wen, X: Conjugate gradient-boundary element solution to the Cauchy problem for Helmholtz-type equations. Comput. Mech. 31(3-4), 367-377 (2003)

5. Marin, L, Lesnic, D: The method of fundamental solutions for the Cauchy problem associated with two-dimensional Helmholtz-type equations. Comput. Struct. 83(4-5), 267-278 (2007)

6. Reginska, T, Tautenhahn, U: Conditional stability estimates and regularization with applications to Cauchy problems for the Helmholtz equation. Numer. Funct. Anal. Optim. 30, 1065-1097 (2009)

7. Cheng, J, Hon, YC, Wei, T, Yamamoto, M: Numerical computation of a Cauchy problem for Laplace equation. Z. Angew. Math. Mech. 81, 665-674 (2001)

8. Denisov, AM, Zakharov, EV, Kalinin, AV, Kalinin, W: Numerical methods for some inverse problems of heart electrophysiology. Differ. Equ. 45(7), 1034-1043 (2009)

9. Kozlov, VA, Mazya, VG, Fomin, AV: An iterative method for solving the Cauchy problem for elliptic equations. Zh. Vychisl. Mat. Mat. Fiz. 31(1), 64-74 (1991)

10. Hao, DN, Lesnic, D: The Cauchy problem for Laplaces equation via the conjugate gradient method. IMA J. Appl. Math. 65, 199-217 (2000)

11. Kabanikhin, SI, Karchevsky, AL: Optimizational method for solving the Cauchy problem for an elliptic equation. J. Inverse III-Posed Probl. 3(1), 21-46 (1995)

12. Qian, Z, Fu, CL, Li, ZP: Two regularization methods for a Cauchy problem for the Laplace equation. J. Math. Anal. Appl. 338(1), 479-489 (2008)

13. Qian, A, Xiong, X-T, Wu, YJ: On a quasi-reversibility regularization method for a Cauchy problem of the Helmholtz equation. J. Comput. Appl. Math. 233(8), 1969-1979 (2010)

14. Tuan, NH, Trong, DD, Quan, PH: A new regularization method for a class of ill-posed Cauchy problems. Sarajevo J. Math. 6(19)(2), 189-201 (2010)

15. Qian, Z, Fu, CL, Xiong, XT: Fourth-order modified method for the Cauchy problem for the Laplace equation. J. Comput. Appl. Math. 192(2), 205-218 (2006)

16. Shi, R, Wei, T, Qin, HH: A fourth-order modified method for the Cauchy problem of the modified Helmholtz equation. Numer. Math., Theory Methods Appl. 2, 326-340 (2009)

17. Fu, CL, Feng, XL, Qian, Z: The Fourier regularization for solving the Cauchy problem for the Helmholtz equation. Appl. Numer. Math. 59(10), 2625-2640 (2009)

18. Qian, A, Mao, J, Liu, L: A spectral regularization method for a Cauchy problem of the modified Helmholtz equation. Bound. Value Probl. 2010, Article ID 212056 (2010)

19. Tuan, NH, Trong, DD, Quan, PH: A note on a Cauchy problem for the Laplace equation: regularization and error estimates. Appl. Math. Comput. 217(7), 2913-2922 (2010)

20. Berntsson, F, Eldren, L: Numerical solution of a Cauchy problem for the Laplace equation. Inverse Probl. 17, 839-853 (2001)

21. Chakib, A, Nachaoui, A: Convergence analysis for finite-element approximation to an inverse Cauchy problem. Inverse Probl. 22, 1191206 (2006)

22. Klibanov, MV, Santosa, F: A computational quasi-reversibility method for Cauchy problems for Laplaces equation. SIAM J. Appl. Math. 51, 1653-1675 (1991)

23. Takeuchi, T, Imai, H: Direct numerical simulations of Cauchy problems for the Laplace operator. Adv. Math. Sci. Appl. $13,587-609$ (2003)

24. Marin, L, Elliott, L, Heggs, PJ, Ingham, DB, Lesnic, D, Wen, X: BEM solution for the Cauchy problem associated with Helmholtz-type equations by the Landweber method. Eng. Anal. Bound. Elem. 28(9), 1025-1034 (2004)

10.1186/1029-242X-2014-19

Cite this article as: Nguyen and Tran: Recovery the interior temperature of a nonhomogeneous elliptic equation from boundary data. Journal of Inequalities and Applications 2014, 2014:19

\section{Submit your manuscript to a SpringerOpen ${ }^{\ominus}$ journal and benefit from:}

- Convenient online submission

- Rigorous peer review

- Immediate publication on acceptance

Open access: articles freely available online

- High visibility within the field

- Retaining the copyright to your article 